STUDII

\title{
« HONTE À L'ENSEIGNEMENT JURIDIQUE AMÉRICAIN » : À LA LUMIÈRE DES LECTURES D'ALAN WATSON
}

\author{
DOI:10.24193/SUBBiur.62(2017).3.1 \\ Published Online: 2017-09-30 \\ Published Print: 2017-09-30
}

Bjarne MELKEVIK*

\begin{abstract}
Shame on American Legal education»: An Critical reading of Alan Watson. The author takes advantage of reading Alan Watson's book "Shame on American Legal Education" to reflect on what is happening today in iusfacultaire culture. On the basis of Watson's assertion that American legal education is shamefully bad and deficient, the author examines successively the false Socratism, which today, in an anti-pedagogical way, undermine the teaching of law; the lure of the case-book method and the legal incompetence that is lodged there; and the phenomenon of poorly trained professors and shoddy research that are becoming more and more widespread. The author deplores the fact that a new legal anti-culture has taken root in the shortcomings and that there is a rampant stupidisation of legal education. He takes a stand in favor of a legal education promoting intelligence and rationality, which takes seriously the demands of modern law and above all its quality, and who can manage the indispensable dialogue between theory of law and practice of law.
\end{abstract}

Keywords: Legal Education; Legal Formation; Legal Didactics; Law Schools; Legal Theory, Philosophy of Law.

C'est par un heureux concours de circonstances que notre attention a été conduite vers le livre "The Shame of American Legal Education » ["Honte à l'enseignement juridique américain "] d'Alan Watson ${ }^{1}$. Publié hors du circuit ordinaire et surtout promotionnel des livres juridiques académiques, rien ne nous indiquait que le livre existait : il était hors radar, dans les limbes de " ce dont on ne parle pas".

La raison se situe dans ce que suggère, sans aménagement, le titre, à savoir que : l'éducation juridique américaine est honteusement mauvaise et déficitaire ou en tout cas, surestimée. C'est, en effet, tout ce que déplore et critique Alan Watson dans son ouvrage. Ses réflexions énergiques et solides s'appuient sur des années d'expérience, en tant que professeur de droit, dans des law-schools américaines, ce qui nous interpelle fortement puisque cela exige une prise de conscience et l'adoption d'une position engagée et non 
ambiguë. Autrement comment être professeur de droit sans s'interroger sur ce que nous faisons et sur ce qui prend tout son sens dans une telle fonction, dans une telle vocation ? Ne faut-il pas, énergiquement, se questionner sur ce qu'il est en, réellement, dans le petit monde de l'enseignement juridique, ne fut-il qu'états-unien ? Et, à ce titre, ne convient-il pas de se rappeler cette petite vérité irritable, qui veut que ce soit incontestablement aux États-Unis que se révèle souvent, en premier, ce qu'attend "patiemment " le reste du monde ${ }^{2}$ ? Au vu de tout ceci, notre objectif, dans cet essai, sera d'examiner, d'analyser et d'expliquer, de façon critique, les propos d'Alan Watson ${ }^{3}$, de même que de réfléchir, en tant que professeur, juriste et iusphilosophe, sur les leçons qu'il faudra en retenir.

En toute lucidité, quelle sera notre réaction face à la "marchandisation », la " mondialisation » (" globalisation ») et la "culturalisation » (" multiculturalisation », " pluralisation ») galopantes, pour ne rien ajouter quant à la « crétinisation » des Facultés de droit et de l'enseignement du droit ? Ne faut-il pas se montrer quelque peu sceptique et critique à l'égard de l'avenir qui nous attend ${ }^{4}$ ? Comment réagir à l'égard des résultats de recherches juridiques avancées qui ne valent que des pacotilles ${ }^{5}$ ? Et le fait de se poser de telles questions ne montre-t-il pas qu'aujourd'hui, tout est loin d'être rose dans les Facultés de droit ? Est-il utile de paraphraser Shakespeare et " Hamlet » et dire qu'il y a, de toute évidence, quelque chose de pourri aux royaumes iusfacultaires ${ }^{6}$ ?

Ceci étant dit, nous pouvons désormais focaliser notre attention sur les thèmes centraux de la « Honte à l'éducation juridique américaine » d'Alan Watson ${ }^{7}$. Dans un premier temps, nous débuterons par une analyse, en profondeur, de ses critiques quant aux enseignements juridiques américains, de même que de l'idéologie langdellien, le platonisme formaliste, le faux socratisme et l'impasse pédagogique. Ensuite, dans un second temps, nous nous pencherons sur sa critique de la "case-book method" et de l'anti-pédagogie qui s'y loge et, surtout, sur la question de la " méthodologie " juridique. Enfin, dans un troisième temps, nous analyserons la situation désastreuse relative à la formation des professeurs de droit et aux écrits "juridiques" de pacotille. Si ces trois "critiques " se veulent franches et directes, $c^{\prime}$ est pour mieux considérer et problématiser ce qui se fait réellement dans les law-schools dominantes et de références ${ }^{8}$.

\section{La critique de l'idéologie langdellien et le faux socratisme}

C'est sur un diagnostic réaliste que se fonde la critique d'Alan Watson. II constate, tel un clinicien, que l'enseignement dans les facultés de droit aux États-Unis se révèle être, grosso modo, lamentable, mauvais et contre-productif. II constate également que cet enseignement en classe souffre d'un manque de systématicité et de logique rationnelle, qui nuit aux étudiants ${ }^{9}$. C'est l'idéologie langdellien et le faux socratisme, ayant mis le grappin sur l'enseignement du droit aux États-Unis, qu'Alan Watson critique et rejette, en ce que ces méthodes sont pédagogiquement néfastes et inappropriées.

Afin de mieux comprendre la critique de Watson, il convient d'abord d'analyser l'idéologie langdellien et le platonisme formaliste, avant de s'attarder, par la suite, sur le modèle du faux socratisme en tant qu'anti-pédagogie qui « plombe » l'enseignement du droit. 


\section{L'idéologie langdellien et le platonisme formaliste}

L'idéologie langdellien représente aujourd'hui le modèle de pensée (et d'enseignement) structurant d'une Faculté de droit états-unienne. L'avènement et la consolidation victorieuse de cette idéologie se démarquent, sans conteste, comme le moment fort pour comprendre l'histoire et les différentes transmutations intervenues dans l'enseignement du droit aux États-Unis depuis la fin du dix-neuvième siècle. Christopher Columbus Langdell $(1826-1906)^{10}$ l'impose, en tant que doyen à la Faculté de droit de I'Université Harvard (poste qu'il occupe de 1870 à 1895), par une réforme conjecturale en profondeur ${ }^{11}$.

Comme le rappel Watson:

Cela a été introduit par Christopher Colombus Langdell, doyen de la Harvard Law School, qui croyait que le droit était une science, et devrait être enseigné dans une méthode scientifique de cas. (...) Le résultat est absurde. Mais la tradition, une fois établie, n'est pas facilement délogée. ${ }^{12}$

En effet, Christopher Columbus Langdell rejetait âprement le modèle pédagogique de l'enseignement juridique de l'époque, qu'il blâmait d'être trop magistral (et doctrinaire). II préférait un modèle nouveau et " scientifique » de l'enseignement du droit supposé (plutôt à tort) être plus professionnel et plus «clinique ». II va l'imposer, remplit de la croyance personnelle que le " droit » n'est finalement qu'une science comme une autre et que rien ne l'en distingue véritablement.

Enseigner le "droit " se révèle, imagine Langdell, identique à un processus de découverte scientifique (c.-à-d. logique et inductif), où les étudiants doivent " repérer » en classe « le droit » en particulier, afin de mieux le comprendre en général. Tel un professeur de chimie qui laisse librement ses étudiants faire des expériences en toute confiance (et n'interviendra qu'en cas de « danger »), le professeur de droit doit agir de même, en laissant aller les étudiants et observer où cela les mène. Le résultat d'une telle séance d'expérimentation devrait à la fin, croyait Langdell, se résumer dans la découverte, supposément, d'un droit (sic) « en général » (ou en idée platonicienne " formaliste »). En tout cas, "le droit » va se manifester, pour celui qui travaille, sous le mode d'un succès clinique et pareil à un succès " en classe ".

Langdell croyait profondément que l'enseignement juridique consistait à imiter scrupuleusement l'exemple des sciences naturelles, des sciences dures, en vue d'obtenir des résultats tangibles et quantifiables. Si on suit son raisonnement, le modèle d'enseignement doit être analogue au travail en laboratoire ou à celui s'effectuant sur un chantier de construction, avant d'être transposé, abstraitement, vers la salle de classe. L'analogie lui suggère en effet que, si les sciences naturelles s'enseignent dans un laboratoire, l'enseignement du droit devrait suivre son exemple puisqu'il ne s'agit que de travailler sur des " choses jugées ", sur des " cas » (c.-à-d. à partir d'une sélection d'abrégés issus du répertoire jurisprudentiel; nous y reviendrons), le tout en vue de reconstruire, en principe 
(i.e. en idée platonicienne-formaliste), le droit qui « est ». Ce qu'atteste le ciel platonicien (en dévoilant intégralement autant l'état « réel » du monde que le " droit » existant) trouve, de ce fait, sa légitimation dans sa force et son efficacité. C'est justement la même " force et efficacité » qu'Hans Kelsen a affirmée dans ses écrits, à partir des années $1920^{13}$.

Langdell s'avère être un partisan convaincu du "positivisme platonicien " (ou formaliste ${ }^{14}$, ou encore de formalisme platonicien), une position largement dominante dans le milieu juridique " formaliste » de l'époque (et même aujourd'hui, surtout chez les constitutionnalistes). Une position platonicienne qui l'encourage à croire que toutes les sciences se rejoignent, sans distinction et sans différenciation, dans le professionnalisme et la technique. Et surtout, il déduit, à tort selon nous, qu'il n'existe pas un " professionnalisme » vraiment propre au "droit " ni à la science " juridique ", sinon un professionnalisme en général, au-dessus de toute spécificité juridique et, surtout, opposée à l'autonomie du droit. Et le dernier nous rappelle que la lutte contre l'autonomie du droit (en pratique et en théorie) ne date pas d'hier et a de multiples sources idéologiques.

À le suivre, l'étudiant doit (supposément) apprendre "le droit " en analysant le droit de manière abstraite (dans la singularité de ce qui a été effectivement jugé ${ }^{15}$ ) qu'il doit comprendre (en principe par induction ${ }^{16}$ ) " en gros »; et le tout, sans jamais recevoir un quelconque enseignement sur la manière dont s'effectue adéquatement une analyse de manière générale. Langdell, de toute évidence, ne se rend pas compte qu'une opération singulière d'induction, opérationnalisée en tant que prémisse d'un enthymème (ou syllogisme tronqué ${ }^{17}$ ) est irrémédiablement fausse, illogique et irrationnelle, et que le saut du " petit » vers le "grand" ne peut rationnellement jamais se réaliser sous le mode d'une "compréhension " ou d'une "connaissance ", ni au singulier ni dans une "généralité " supposée ${ }^{18}$. En réalité, aucune induction ne mène à la " connaissance ». Un cas singulier, de même qu'une multitude de cas jugés, ne peut conduire à pouvoir en faire une " généralité ". De plus, il ne s'agit en rien d'un processus permettant de découvrir ce « qu'il en est réellement ». Nonobstant les croyances de Langdell, le prétendre serait une tricherie intellectuelle (et morale) et, pire encore, cela ne nous permet pas d'arriver à comprendre comment notre psychologie peut se jouer de nous pour mieux nous desservir, à regret ${ }^{19}$.

C'est rationnellement et logiquement impossible car, en effet, il n'y a rien dans le " petit » (c.-à-d. dans la " chose jugée » et dans un texte jurisprudentiel) qui permet de remplir le vide entre le " petit » et le " grand ». Personne ne peut non plus prétendre être capable de sauter hardiment vers une conclusion en utilisant un terme, si ce dernier ne lui a jamais été expliqué. On ne peut donc valablement pas porter d'affirmation sur « le droit en général » par " un droit » au singulier, sorti de nulle part. C'est en effet insensé et irrationnel, d'autant plus que tout cela se joue et se déjoue à l'intérieur de la psychologie individuelle et à la merci même de cette psychologie. Logiquement, l'induction à partir des " choses jugées » nous conduira généralement nulle part, sauf vers un retour des « choses jugées » et sans recevoir de concept supplémentaire ! Le fait de croire qu'il existe une "logique unique», une nécessité logique, qui viendrait nous imposer une "cause" explicative (i.e. une cause conceptuelle quelconque, ou spécifiquement la révélation de la 
cause et du concept du « droit »), relève clairement de l'irrationnel, de la manipulation, ou encore des dédales insondables de notre propre univers psychologique.

L'analyse d'un texte de la " chose jugée » est, insiste Alan Watson, logiquement et pédagogiquement " mutilée " si aucune explication systématique et rationnelle n'est développée ou produite raisonnablement, en toute indépendance, en se demandant «pourquoi $»^{20}$. Et pour cela, il convient non seulement de s'interroger intellectuellement sur le sens juridique du "pourquoi » mais, également, de s'attarder sur l'importance du fait qu'elle soit " à part » et logiquement là en tant que doctrine juridique.

Approfondissons l'argument et retenons-en qu'il est irrationnel de fermer les yeux sur tout ce qui est contextuellement " sous-entendu " dans une analyse d'une " chose jugée » ( $c^{\prime}$ est le cas lorsque les jugements ne sont pas soigneusement analysés en tant que cas particuliers et n'ayant de sens qu'à l'intérieur d'un ordre judiciaire en soi problématique et " sous enquête »). II est en effet absurde de ne pas analyser la jurisprudence au niveau même de sa construction et de sa structure, en tant que telle et à l'égard d'un ratio decidendi que le praticien (autant que le théoricien) construit pour donner un sens, que ladite jurisprudence (et toute jurisprudence) ne possède pas en soi. Cela appauvrit irrémédiablement la compréhension qu'un étudiant peut se faire de son rôle de futur juriste. II ne pourra pas le comprendre, si personne ne le lui a jamais appris. Il s'agit là d'un des rôles de la doctrine juridique en tant que "fournisseur de sens "; on comprend alors toute la responsabilité qui pèse sur ses épaules !

A contrario, sans explication et sans " mise en contexte ", tout exercice langdellien d'un abrégé jurisprudentiel flotte en air, pareil à une métaphysique qui prendrait la forme d'un nuage changeant chaque milliseconde. Si une personne ignorante peut avoir l'impression d'avoir compris, le fait est que c'est justement cette ignorance dans la « compréhension » qui le condamne à ne pas comprendre, voire même à ignorer, qu'il n'a pas compris.

Le "faux socratisme " des law-schools, accentuons-le, ne cherche ni l'universel, ni le " conceptuel ", ni la rationalité juridique et ni, non plus, la rationalité d'une pratique juridique au service des clients. II n'interroge en rien le domaine propre de la rationalité à l'égard du juridique, il ne mobilise pas l'histoire, la science, la société ou encore la pratique du droit pour mieux, adversa sententǐa, s'illusionner à l'égard des idées platoniciennes qui sont supposées être disponibles via la révélation, grandeur nature, des principes et des règles de droit (et tout ce qui va avec!). Rien de bon n'en sortira !

Alors, quand Alan Watson nous enseigne qu'en l'absence d'un enseignement synthétique (et rationnel) les étudiants ne recevront guère autre chose qu'un amas d'abrégés éparpillés de jugements sélectionnées et écourtées infidèlement et idéologiquement par chaque professeur, il a raison. Ce qui est irrationnel, c'est de vouloir nourrir le peuple en distribuant des pierres, plutôt que du pain ${ }^{21}$ ! Sans une explication doctrinale actualisée pour éclairer, guider et expliquer rien ne peut être compris, avec responsabilité. Combien d'étudiants en droit saisissent alors que le modèle langdellien nuit à la compréhension du droit et à la formation d'un esprit juridique critique et réfléchi et que, in fine, cela nuit à leur formation en tant que juristes? 


\section{Le faux socratisme et le désarroi étudiants}

L'idéologie langdellien se targue d'être "pédagogique " et " socratique ", là où Alan Watson (et nous agréons) ne voit qu'un modèle élitiste (incontestablement eugénique), une célébration de l'univers platonicien et des idéaux-sophismes concernant le " droit ». C'est, indique-t-il, une anti-pédagogie bâtie sur la sophisterie et sur une idéologie qui trompe les étudiants en les laissant désarmés et bouche bée devant la complexité et les difficultés des raisonnements juridiques (nécessairement complexes) ${ }^{22}$. Surtout, c'est une forme d'enseignement en hauteur qui abandonne les étudiants à eux-mêmes, car personne ne leur explique adéquatement et systématiquement le " sens du droit à construire ", qui est en jeu dans un cas juridique spécifique, de même qu'en général. II s'agit là d'une tendance. Or, de quelle sorte de " pédagogie socratique " parlons-nous ? Ne s'agit-il pas, suggère Alan Watson, que d'une imposture et d'un faux socratisme anti-pédagogique ? À cet égard il écrit :

La méthode socratique. La méthode dite socratique de l'enseignement - un abus de langage en fait - est endémique aux États-Unis. (........) Ceux qui l'utilisent - la grande majorité des professeurs de droit - croient qu'ils aiguisent les compétences analytiques. Dans une large mesure, je suis en désaccord : c'est l'enseignement par la terreur. Le professeur prend, habituellement apparemment au hasard, un étudiant qu'il questionne sur une affaire donnée. L'étudiant peut rarement répondre de manière satisfaisante. ${ }^{23}$

Alan Watson insiste fortement sur les dégâts psychologiques (et moraux) qu'une telle posture provoque chez les étudiants. Comme il le souligne, une fois en classe la séance langdellien devient, pour l'étudiant en droit, un acte de soumission qui oscille entre la terreur et l'apathie, où le plus important sera de se "sauver " plutôt qu'apprendre. Dans ce climat de terreur, celui ou celle qui sera soumis à l'interrogation "fausse socratique " souffrira et endurera, tandis que celui qui aura l'occasion d'y échapper s'évadera psychologiquement à l'intérieur de lui-même ${ }^{24}$. D'où la stratégie estudiantine de " fermer les oreilles "sur ce qui se passe en classe, pour mieux se préparer psychologiquement quand ce sera à son tour d'être malchanceux ${ }^{25}$.

$\mathrm{Au}$ niveau pédagogique, une telle logique de terreur ne permet à personne de comprendre, en profondeur, le domaine juridique: c'est-à-dire ni ses finalités, ni ses techniques et ni, non plus, le sens moderne du droit. Et comme il est vrai que les étudiants ne peuvent rarement répondre adéquatement, la logique du "questionnement " à l'aveugle et sans guidage sera forcément un exercice "inquisitoire " où le coupable se devra d'avouer qu'il n'a rien compris et rien appris pour, en fin de compte, ne recevoir qu'un sourire hautain d'un professeur qui ne daignera pas donner d'explication. C'est une anti-didactique, une anti-pédagogie, qui se moque des étudiants et qui refuse de les aider à apprendre et à comprendre le domaine juridique. Dans ce cas, comment agir, penser et procéder à l'égard d'une question de droit ? Comment arriver à comprendre si aucune explication n'est donnée ? Ou encore, comment être sûr d'avoir compris, si cela ne peut jamais être confirmé d'une manière ou d'une autre ? Comment apprendre à nager, si on ne vous permet que de flotter? 
Une conséquence de cette façon d'enseigner c'est que, de manière pédagogique, l'apprentissage du droit ne pourra jamais se réaliser dans les amphithéâtres ou dans les salles de classe. Comme ils ne sont pas à ce point stupides, les étudiants vont rapidement se rendre compte que le plus important, ce n'est pas l'étude du droit en classe, mais bien la nécessité (presque animalière, atavique et "darwinien ») de survivre, de ruser et de se prémunir contre le risque de se ridiculiser lors des séances langdellien de faux socratismes. Dans cet acte de " prévision », c'est l'apprentissage en privé qui sauve les " bons » étudiants. On va pouvoir, de ce fait, distinguer ceux qui sont prêts à s'investir pour survivre, des autres. D'où le rôle inattendu (et le trafic) des notes d'étudiants des cours des années précédentes et les manuels lus en cachette et en "étude libre ", sans guidage et sans explication professorale. Et quant aux " anciennes notes de cours ", les étudiants qui viennent d'un milieu aisé (et d'alumni juridique) auront tous les avantages que les autres étudiants qui viennent d'un milieu modeste (et qui n'auront pas pu se renseigner sur les modes de survie) n'auront jamais.

Le résultat de l'idéologie langdellien c'est qu'aucune explication claire quant « au sens du droit » n'est fourni aux étudiants. À aucun moment, ils ne recevront un éclaircissement rationnel et un développement doctrinal explicatif aptes à leur faire comprendre comment tout ceci fonctionne juridiquement et comment cela peut prendre tout son sens en pratique. Ils ne verront pas quels sont les vecteurs logiques et argumentatifs qui sont mis en œuvre dans le droit moderne et de la même manière, ils ne pourront jamais savoir comment mobiliser adéquatement l'expérience accumulée qu'un ordre juridique collige dans sa jurisprudence.

Comme le remarque Alan Watson:

Imaginez donc un cours universitaire de physique où seules quelques expérimentations isolées font cas d'études, où le lien entre l'un et l'autre n'est pas expliqué, où les fondements théoriques ne sont pas présentés et où la quasi-totalité des écrits des spécialistes sont ignorés. Le résultat est absurde. ${ }^{26}$

Absurde en effet ! Facile de lui donner raison ! Or, n'est-il pas étrange de ne s'en tenir (c'est le cas pour Langdell) qu'au seul critère (pourtant non défini et non spécifié) de " succès en classe " ? Également, n'est-il pas absurde de soutenir que la qualité et la vérité emporteront toujours la victoire sans qu'il soit nécessaire de faire quoi que ce soit pour vérifier cela ? L'alternance pédagogique (et didactique) d'une bonne et d'une mauvaise réponse est pourtant clairement exclue, car une telle méthodologie n'est pas considérée comme étant réellement pertinente. Pour cela, il faudrait que le professeur daigne expliquer le " pourquoi ", le " comment » et la " raison et la logique " d'un raisonnement juridique de qualité. À savoir : pourquoi une mauvaise réponse est "mauvaise " et une bonne réponse " bonne » et, surtout, comment faire pour leur " donner du sens " dans le domaine juridique; ce que ne permet jamais le faux socratisme de Langdell.

Or, si c'est uniquement le succès en classe qui prévaut et qui compte, le caractère pratique (et même évaluatif) du droit échappe à la compréhension, précisément parce 
que la logique de répétition (rappelant nos propos sur les limites de l'induction) n'ouvre pas la porte à l'innovation ou à la maîtrise de ce qui peut être fait dans le domaine juridique. Si le critère de Langdell n'était que l'horizon d'un "succès en classe ", on pourrait l'illustrer par des aveugles qui guident les aveugles en se réjouissant d'avancer ensemble! De ce fait, le " faux socratisme » n'exploite-t-il pas, ici, une faille psychologique chez l'individu moderne et, plus spécifiquement, sa propension à chercher le juridique dans la rêverie qu'inspire le mot "droit " ? Une faille psychologique où, tout en hissant indument le drapeau de "la critique, de l'ouverture et du pluralisme " (les mots magiques que trahissent régulièrement les partisans du Ligue du politiquement et moralement correct, du pluralisme idéologique ou du post-post), il ne s'agit, en fin de compte, que d'éviter à tout prix de faire des critiques, de faire des vagues et, surtout, d'éviter des interrogations pertinentes et utiles sur notre façon de faire le droit. Le futur ne sera alors que business, management, gouvernance, développement durable et dos courbés.

\section{Critique contre le leurre non tenu des « recueils d'arrêts "}

Examinons maintenant, avec Alan Watson, l'inculture des " case-book » et leur rôle anti-pédagogique ${ }^{27}$. C'est la conséquence logique et rationnelle découlant de la critique précédente, contre le faux socratisme et la démission professorale. II juge, en effet, que la case-book methodest corruptrice et mauvaise autant pour les étudiants que pour les professeurs. C'est pour lui une « inculture » qui, sans portée pédagogique réelle, n'apporte rien de bon et ne sert finalement qu'à rendre l'esprit plus mou et manipulable. II s'agit là d'une inculture, d'une anti-pédagogie, d'une non-méthodologie et d'une futilité qui trompe et déroute les étudiants et dont il faut se débarrasser dans la mesure du possible. Regardons donc, de plus près, les arguments qui appuient ces jugements.

\section{Une méthode néfaste d'anti pédagogie}

Soulignons que, pour Alan Watson, les « case-books " sont mal faites, car elles sont concoctées sans expliquer le sens juridique qui est engagé dans la " chose jugée » et sans qu'une analyse quant au fonctionnement réel et pratique du droit ne soit fournie. Les " case-books » entrent, de ce fait, dans une anti-pédagogie aveuglante, puisqu'il n'existe aucun enracinement dans cette pratique contextuelle problématisée. De plus, elle ne fournit aucune explication pouvant ajouter de la matière à cette pratique. L'appréciation d'Alan Watson est subséquemment sans appel. En effet, une large part de l'ouvrage " Honte à l'enseignement juridique américaine " sert à illustrer en détail pourquoi les "casebooks " sont mal faites et pourquoi il y a tant de raisons de s'en méfier pour, in fine, arriver à se défaire complètement de ce système.

Alan Watson déplore, avant tout, l'effet néfaste des "case-books » relatif à la formation juridique qui, au lieu d'éveiller l'intelligence juridique, n'est en fin de compte qu'une anti-doctrine problématique: 
"L'approche du recueil américain (...) est confuse et peu informative. Les cas individuels sont édités abrégés et arrachés de leur contexte. Les étudiants ne sont pas informés si l'affaire est importante pour la compréhension du droit ou est plutôt périphérique. Ils ne reçoivent pas non plus un aperçu des grands principes de la question. Puisque c'est l'approche standard dès la première journée dans l'école de droit, les étudiants de première année deviennent paranoïaques. Les étudiants de deuxième année s'ennuient, un constat reconnu comme tel par la quasi-totalité des professeurs de droit. $"^{28}$

S'observent clairement, accentue Alan Watson, des «case-books » remplies d'abrégés et de cas amputés et mutilés, qui ne sont jamais présentés intégralement. Ceuxci vont plutôt osciller entre une courte présentation (une version de quelques lignes), une longue citation (une version d'une demi-page) ou une version de quelques pages ${ }^{29}$. On retrouve également des abrégés présentés en vrac, sans aucun soin et sans aucune logique sur le plan juridique. Il s'agit, souligne Alan Watson, d'un pêle-mêle artistique, audelà de tout critère de systématicité et de rationalité, où le professeur faux socratique " ne s'implique pas " véritablement avec les étudiants et, surtout, où il joue son propre jeu. Dit autrement, on est face à un jeu de pouvoir où le professeur prend « soin » de sélectionner ces abrégés parce qu'ils sont " singuliers ", " exotiques » et " littéraires » ou encore parce qu'ils sont affectionnés (pour de mauvaises raisons ?) par un des composants de la Ligue du politiquement correct. Or, cette idée ambitieuse de résumer, par exemple en abrégé, les " choses jugées" dans le domaine de la " responsabilité civile " par quelques décisions judiciaires sélectionnées arbitrairement et sans jamais daigner donner d'explication adéquate, peut-elle avoir une quelconque valeur ou encore un sens rationnel sur le plan juridique ? Ou est-ce que cela frôle plutôt l'absurdité et l'irrationalité tant ceci manque cruellement de portée logique et pédagogique ? Ceci, d'autant plus, que ces abrégés sont imposés comme seule lecture obligatoire pour les étudiants en première année de droit. Ils sont donc adressés à des personnes qui n'ont pas assez d'information et de formation pour arriver à les évaluer de manière adéquate en tant qu'autorités pouvant, éventuellement, intéresser le milieu juridique. De plus, si ces abrégés sont fréquemment accompagnés de commentaires littéraires et d'interpolations idéologiques propres au " club d'identification » auquel adhère personnellement (et spéculativement) le professeur, une telle éventualité juridique peut-elle être rationnellement actualisée au profit du droit ?

Comme le souligne Alan Watson, les « cases books " ne permettent nullement de comprendre comment fonctionne le droit parce qu'elles fonctionnent avant tout comme une mise en scène, presque théâtrale et "dramatique ». Une mise en scène où une idée non expliquée s'affirme, artificiellement, en tant que "droit " pour mieux prendre vie, tel un jeu d'ombre et de lumière oscillant entre art et littérature, entre idéologie et mode, entre virtuosité narrative et imposition psychologique. Alan Watson ne voit simplement aucune vertu pédagogique dans la culture des "case-books". II ne les perçoit guère autrement que comme des « recueils d'arrêts » fourretouts, des copiés-collés jurisprudentiels 
" sans queue, ni tête " ou encore bourrés " d'extraits " chaotiques (et exotiques) reproduits sans explication selon les préférences dites du « milieu » ou, plus plausiblement, selon le cercle idéologique auquel appartient le professeur. Ce sont des livres fourretouts qui n'ont, tout compte fait, rien de didactique ni de méthodique dans le sens commun et pédagogique du terme. Qui plus est, ils semblent pencher dangereusement vers la tromperie et la servilité tant ils ne permettent pas aux étudiants d'avoir une compréhension claire ni de l'état du droit (le droit positif) ni de ce qui peut être fait dans le domaine juridique.

Alan Watson postule aussi qu'il y a une étroite relation entre l'enseignement via la " case book " et la paresse intellectuelle (et morale) des professeurs (qui peut d'ailleurs facilement se conjuguer avec une frénésie facultaire et extrafacultaire, de même qu'avec des surinvestissements idéologiques et "théoriques " sans valeurs certaines). II observe froidement que l'adoption, par un professeur de droit, d'une "case book » réputée va habituellement s'accompagner d'un cadeau, par l'Éditeur (de la case-book), représentant un recueil de réponses recommandables et acceptables (c.-à-d. un recueil non disponible pour les étudiants). L'enseignant n'a donc pas trop à se creuser les méninges ou à faire de la recherche puisqu'il possède le livre des « réponses recommandées ». En effet, il n'a qu'à regarder le livre des "réponses recommandées " qu'on a mis à sa disposition et s'en satisfaire. II n'a plus à maîtriser le domaine qu'il enseigne. Et quant au rédacteur (ou rédacteurs) d'une " casebook » il (ils) n'aura(ont) qu'à attendre patiemment son pactole d'argent.

Ainsi se brise distinctement et tristement le lien pédagogique entre l'enseignement d'un professeur et sa responsabilité, censée œuvrer à la construction de la doctrine, qu'il est supposé maîtriser, écrire et enseigner. C'est une rupture avec le credo voulant qu'en enseignant ce qu'il sait par sa propre recherche (refusant donc d'enseigner uniquement à partir des « livres » ou la « recherche » de quelqu'un autre ${ }^{30}$ ), il se porte garant de l'exactitude, de la pertinence et de la " scientificité » d'une " description » (i.e. un état des lieux) de ce qui compte en tant qu'état juridique d'une question de droit. La culture des "case-books » ne nous révèle donc rien d'autre qu'un modèle cassé où l'enseignement et la recherche sont coupés et séparé l'un de l'autre. Pire l'un des pôles, à savoir la recherche doctrinale, se retrouve marginalisé et sous-évalué, tandis que l'autre pôle, à savoir l'enseignement, a été cédé au modèle de l'autorité et de la soumission. C'est notamment un modèle où la recherche ne se concrétise jamais dans un enseignement de qualité, un modèle où il n'y a plus aucune dialectique entre la recherche et l'enseignement, sinon où la " recherche » a toute l'opportunité de devenir idéologique, spéculative et post-moderne. Dès lors, le temps libéré par la "case-book», pour un professeur, va lui permettre de faire de la recherche de " pacotille » et de le faire avec style. D'où la question de savoir si, libérée de tout contact avec la réalité juridique, la nouvelle recherche utilisant le mot " droit " n'estelle pas finalement une littérature comme les autres ?31

Se construit entièrement la dépendance, en hétéronomie, d'un professeur qui n'a désormais plus le contrôle entier et intellectuel sur son enseignement ou sur une thématique/domaine du droit. À la limite, il ne s'agit guère d'autre chose que d'un professeur qui a lu la "case book» avec son manuel de "réponses recommandées ». 
Comment un tel professeur peut-il inspirer ses étudiants ? Comment peut-il servir pour transmettre une connaissance de droit qu'il n'a pas lui-même ou qu'il n'a pas acquise adéquatement par une recherche individuelle ? Comment peut-il transmettre l'importance de se mettre au service des justiciables, s'il n'est là, lui-même, que dans le rôle d'un exploiteur, d'un imposteur?

La « case-book method » représente aujourd'hui, avant tout, une tradition qui se répète et se perpétue comme si de rien n'était et où personne ne se pose de questions. Les professeurs n'ayant jamais été formés intellectuellement et doctrinalement en vue d'acquérir, de consolider et d'approfondir une compréhension systématique, méthodique et rationnelle du droit, n'ont simplement aucune raison, croient-ils, de se questionner, de s'interroger. En réalité, ils ne font que répéter ce qu'ils ont eux-mêmes subi et enduré, pour mieux le faire perdurer, et ce, sans état d'âme. Le résultat, assure Alan Watson, c'est de faire " comme d'habitude ", de ne " jamais se poser de questions " et de faire (et d'utiliser) des « case-books », comme cela en est devenu la tradition ${ }^{32}$.

Mais alors, pourquoi perpétuer la " case-book method " aujourd'hui ? II n'y a, en fait, dans la " case-book method », ni de véritable transmission de " connaissances » ni de véritable enseignement juridique. II n'y a que ce que l'enseignant, dans son rôle d'animateur de classe, dit à partir d'un livre qu'on lui a donné.

Et si l'idéologie langdellien insiste sur le rôle " méritoire » de l'étudiant et sur sa valorisation en classe afin qu'il trouve " en lui » sa valeur marchande, l'enseignant peut-il représenter autre chose qu'un clown qui plaît, qui amuse et qui divertit, en " droit " ? Celui qui s'y connaît, ne serait-ce qu'un peu, sur le phénomène de "baromètre de popularité » des enseignants de droit en Amérique des Nord entretenu par différents sites électroniques (ou encore par des associations étudiantes), peut devenir quelque peu songeur !

En réalité, Alan Watson suppose qu'il s'agit surtout d'assurer une sorte de darwinisme social (ou " méritoire " selon Langdell), où la sélection à l'oligarchie juridique future se fait par une séparation, une expulsion du plus faible (psychologiquement et socialement parlant) durant la première année. De ce fait, la survie du plus fort va lui assurer à la fois une force et une solidité du système juridique en tant que piliers pour l'avenir. Le rôle particulier (et si sociologique) qu'a joué le droit ou plus précisément l'oligarchie juridique, entre le $19^{\mathrm{e}}$ et le $21^{\mathrm{e}}$ siècle, pour stabiliser la société états-unienne, en l'absence d'autres vecteurs sociaux forts, peut soutenir ce point de vue. Mais, n'est-il pas temps de rappeler que le droit ne doit pas uniquement servir le recrutement à l'oligarchie juridique (qu'il soit de gauche ou de droite), et envisager que peut-être que le droit doit, avant tout, servir celui qui a vraiment besoin de l'assistance juridique et surtout de la représentation juridique?

\section{Une non-méthode de futilité et d'alternative}

Nous l'avons affirmé, pour Alan Watson la méthode des case-books représente avant tout une non-méthode et il convient, en conséquence, d'analyser plus en détail cette appréciation sur le plan pédagogique, au niveau de sa futilité. Pourquoi, en effet, entamer un 
régime pédagogique d'inquisition en 1re année de droit pour, par la suite, le prolonger sur deux autres années ennuyeuses et monotones ? À quoi cela sert-il pédagogiquement ? À suivre Allan Watson, cela ne mène à rien, et rien ne bouge à cause de la tradition langdellien. Pédagogiquement ce n'est, répétons-le, qu'une " méthode de futilité » :

La méthode de recueil d'arrêts est en fait un exercice de futilité. Ce sont les étudiants eux-mêmes qui sont censés se construire une image du droit à partir de bribes généralement déconnectées qui leur sont disponibles, et ce, virtuellement sans outil. Les étudiants doivent se débrouiller pour deviner ce qu'est le point de vue de l'éditeur quant à la loi, plutôt que d'approfondir ce qui est en jeu juridiquement. Au lieu d'étudier les arguments de l'arrêt à la lumière du raisonnement de la pensée conceptuelle qui l'a précédé et de sa place dans un réseau de principes structurés, on leur fournit, dès le départ, une seule instance qui s'autojustifie seulement en référence à des caractéristiques particulières, laissant beaucoup à comprendre. Parce que basés sur des principes établis, plusieurs aspects cruciaux de l'arrêt ne sont pas pris en compte ni mentionnés. (...). Le problème s'aggrave lorsque la discussion se limite en grande partie à la notion "Cela est-il juste ? ${ }^{33}$.

Face à une telle question, notre réponse sera plutôt : bien sûr que non ! II n'y a rien de " juste » là-dedans ! Une futilité qui trompe, qui dompte et qui amène l'étudiant vers le néant, n'a pas de valeur pédagogique du tout. Il faut se distancier et chercher une alternative; ce qu'Alan Watson esquisse ainsi :

Mon point n'est absolument pas que l'apprentissage du droit serait beaucoup plus facile - il le serait évidemment - si les concepts et les règles développés dans les exemples de cas avaient d'abord été exposés brièvement, avant d'être suivis par une discussion de quelques cas choisis pour illustrer les règles, les paramètres et les questions soulevées. C'est quelque chose de nécessairement souhaitable. C'est peut-être une vertu que certains de ses collègues pensent à faire une étude de droit de première année inutilement difficile. Mon point est que l'approche standard déforme la manière dont le droit est, comment il se développe, et sa relation avec la société. Les concepts et principes sont minimisés. Ainsi sont les règles, et leur autorité et la stabilité. Les cas sont retirés de leur contexte juridiquement pertinent et l'autorité du contexte est démantelée. Curieusement, peut-être, un autre résultat de cette approche est que les cas sont souvent retirés de leur contexte social. (...) L'absence de fondements théoriques est une erreur fatale dans la démarche du recueil. ${ }^{34}$

Alan Watson cherche, en effet, une pédagogie variée du droit, une pédagogie du droit qui explique, autant que possible la manière d'assumer un rôle responsable à l'intérieur du droit, comment maîtriser le système juridique et comment être utile pour la société. Son point de vue est qu'il convient de se mettre à la place des étudiants en droit et de prendre la mesure de la route à suivre, à partir de leurs chaussures. De ce fait, le fonctionnement du droit dans une société moderne s'apprend pas à pas, étape par étape, de même que par une initiation lente et laborieuse. Apprendre et comprendre le 
fonctionnement du droit ne se résout pas dans une quelconque formule magique ou " pluralisée ». Au contraire, tout se fait en pariant sur la dialectique professeur et étudiants, le tout en faveur d'une intelligence juridique en construction. II s'agit de comprendre la complexité juridique en abordant comment le droit a été construit historiquement et dans la jurisprudence. II s'agit, aussi, de comprendre comment fonctionne le système juridique dans son ensemble et en particulier.

Quand, de ce fait, Alan Watson soutient, tel que cité auparavant, que le nonexamen du sens juridique de cas jurisprudentiels (sous le mode d'un abrégé mutilé dans une " case-book ") est à la fois absurde sur le plan juridique et néfaste au niveau pédagogique, il a raison. Les cas jurisprudentiels où le sens juridique n'est pas étudié adéquatement n'assurent jamais une bonne compréhension (ou, pire, favorise une compréhension superficielle et idéologique). L'examen d'un cas jurisprudentiel qui met entre parenthèses le contexte, triche et devient inévitablement contre-productif, car le contexte rime avec l'intelligence, la relevance et la portée juridique. L'examen d'un cas jurisprudentiel qui néglige d'analyser adéquatement la contribution adéquate qu'a pu jouer la législation (et les sources de droit en général) s'aveugle et, pire, devient une arme de l'antidémocratisme que chérit trop une portion de l'iusoligarchie contemporaine.

De manière sous-jacente au débat pédagogique de "comment enseigner le droit ", nous apercevons, en fin de compte, une autre question hautement politique (et démocratique) : le droit doit-il servir "tout le monde " ou doit-il, au contraire, servir pour gouverner, administrer, gérer, politiser, " moraliser », etc. ? N'est-il pas étrange qu'aujourd'hui ce soit le modèle consistant à gouverner les « autres " qui a gagné les esprits postmodernes (autant de gauche que de droite) ? Dans la lutte renversée des classes, la lutte contre le peuple n'est-elle pas la nouvelle "pater-maternalisme » qui submerge dans le désir de voir les « autres » courber leurs têtes?

\section{Les professeurs et la fermeture de l'esprit juridique}

Alan Watson n'est guère plus doux avec les professeurs de droit. Plus précisément, il ne manifeste aucune tendresse excessive à l'égard de la formation intellectuelle et juridique reçue par de nombreuses personnes sélectionnées pour devenir professeurs. II les trouve mal formés, mal instruits, mal éduqués et mal préparés en vue d'assumer adéquatement leur rôle de professeurs de droit. Tout se résume dans un jugement sans appel :

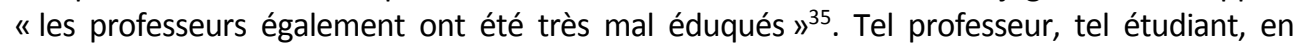
quelque sorte ! Une assertion qu'il s'agit d'expliquer et d'exemplifier plus amplement.

\section{Les professeurs sont très mal éduqués}

L'appréciation critique d'Alan Watson à l'encontre de la culture juridique de plus en plus manquante, voire absente, des professeurs du droit se comprend (pareille à ses autres jugements) sous le mode d'un verdict à l'encontre d'une culture généralisée de laxisme quant à la sélection et la formation des professeurs de droit. Si Alan Watson 
appuie fortement et sans ambiguïté qu'il existe également d'excellents professeurs aux États-Unis, c'est la non-culture montante de la "non-excellence » qu'il critique et qu'il déplore. Il observe (et critique) que la sélection pour devenir professeur de droit repose de moins en moins sur des critères clairs et vérifiables au niveau de la recherche juridique. De plus, il constate que cette sélection va davantage s'appuyer sur l'image commerciale, sur la présélection des nouveaux privilégiés (selon race, ethnie, orientation sexuelle, etc.) et surtout, sur l'adhésion à l'une ou l'autre des branches de la Ligue du politiquement correct. Souvent, les professeurs de droit sont sélectionnés en tant que " personnalité » apte à incarner une image facultaire (inévitablement selon l'oukase de la Ligue du politiquement correct) ou, encore, selon une image prête à séduire des donateurs d'argent comptant. Si les professeurs sélectionnés en savaient davantage sur le droit, cela serait certainement apprécié mais, en fin de compte, cela ne pèsera pas beaucoup dans la balance.

Seront recrutés comme professeurs de droit ceux qui représentent une valeur marchande, une valeur oligarchique, une valeur de séduction. II s'agit d'un schéma de recrutement qui est déjà mis en œuvre dans les cabinets d'avocats, avec les associés et les partners qui peuvent rapporter le plus d'argent avec de riches clients. C'est ce modèle qui s'infiltre de plus en plus dans le monde iusfacultaire (i.e. subventions, allocations, dotations, bourses, donations, etc.).

À suivre Alan Watson - et en simplifiant son argumentation - la crise de l'enseignement juridique, la crise de la méthode pédagogique et la démission pédagogique s'aggrave et s'amplifie à l'intérieur du corps professoral à cause de cette incapacité iusfacultaire de corriger la situation. De plus en plus de professeurs de droit ne sont simplement rien d'autre que des plombiers qui s'ignorent et qui s'auto-estiment, sans raison, en pensant représenter, à eux seuls, des génies du "droit ». Le problème, c'est qu'en l'absence de "doctrine ", par la dévaluation rationnelle du droit, de même que par sa marginalisation intellectuelle et pratique, tout cela aura des conséquences néfastes sur le climat iusfacultaire. II manque simplement, souligne Alan Watson, une relation logique entre le travail effectué par un professeur (concernant une question de droit) et ce qu'il enseigne au bénéfice de ses étudiants (concernant la compréhension à l'égard de cette même question). Sans cette relation dans laquelle le professeur transmet ses compétences concernant le droit, ce dernier parle sans savoir et sans connaître, et il parle sans avoir fait de véritables recherches dans les domaines qu'il enseigne.

En fait, l'avantage d'un professeur ayant une connaissance solide et adéquate de la " doctrine juridique » est normalement la constatation qu'il a obtenu cette connaissance parce qu'il (elle) a mis lui-même la main à la pâte pour faire de la recherche et qu'il a ainsi " mesuré et compris la pratique » du droit ! Un tel professeur acquiert une autonomie en tant que professeur-chercheur véritable, et en tant que savant sérieux, parce qu'il maîtrise la façon d'écrire adéquatement une œuvre doctrinale de qualité. De ce fait, il est capable, dans l'enseignement, d'utiliser la dialectique entre théorie, dogmatique et "pratique » pour enseigner la compréhension et le sens juridique (la doctrine) de manière telle que cela va donner un sens à ce qu'attend, en pratique, un étudiant en droit. 
En contraste, souligne Alan Watson, comment obtenir la même chose chez un professeur qui n'a pas acquis de connaissance véritable du droit et qui est constamment obligé de tricher?

\section{Trois professeurs très mal éduqués}

Le plus long chapitre est consacré à l'examen de la recherche de pacotille de trois professeurs célèbres et influents. C'est ici qu'Alan Watson arrive avec sa critique la plus directe et sans aucune complaisance à l'égard de la bimbeloterie intellectuelle et l'insuffisance béotienne que caractérisent ces trois professeurs, d'ailleurs nommément identifiés ${ }^{36}$. L'objectif servira à couper l'herbe sous le pied de leurs nombreux admirateurs dévoués aux États-Unis (et ailleurs) et à exposer, au grand jour, la profonde ignorance juridique que témoignent leurs écrits. Les trois pontifes se nomment Abraham Arthur Schiller (1902-1977), professeur de droit romain à Colombia University à New York; Morton J. Horwitz (1938), professeur de l'histoire du droit des États-Unis à Harvard Law School ; et Duncan Kennedy (1942) professeur de droit à Harvard Law School.

Dans le dossier "Abraham Arthur Schiller ", Alan Watson - lui-même une sommité en droit romain - s'intéresse au professeur $S$ chiller ${ }^{37}$ et, plus particulièrement, à sa trouvaille (supposément) "révolutionnaire » dans le domaine du droit romain ${ }^{38}$. A. Arthur Schiller est réputé pour avoir trouvé que le " secret commercial » d'aujourd'hui est issu directement du droit romain classique et du droit des esclaves romains ${ }^{39}$. Il défendait, en effet, que la formule d'ester en justice intitulé "Actio Servi Corrupti ", à savoir la possibilité pour un propriétaire d'esclave dans la Rome antique de demander réparation (et de la protection et de l'intégrité corporelle) pour (et contre) l'abus d'un tiers, constitue cette origine. A. Arthur Schiller défendait spécifiquement que cette formule permît d'ester en justice en prétendant qu'une tierce personne avait " rendu l'esclave corrompu ». Une affirmation contre quoi, Alan Watson se révolte, car elle est fantaisiste et non fondée.

À Alan Watson de rectifier et de souligner qu'en histoire du droit antique, il s'agit là, effectivement, d'une actio [i.e. une formule de procédure] qui protège contre la corruption d'un serviteur [un/une esclave], par une tierce personne. Pour autant, cela ne se situe point au niveau de la " propriété " ou du « bien ", sinon entièrement au niveau de la corruption morale et sexuelle d'un esclave pouvant porter préjudice à son propriétaire, à sa famille et à sa maison. C'est une actio qui a subséquemment quelque chose d'essentiel à voir avec l'honneur de la famille et la respectabilité sociale, mais rien, toutefois, avec le « secret commercial ».

Alan Watson indique qu'en associant la protection des secrets commerciaux à celui de la corruption morale et de l'honorabilité que pouvait faire subir une esclave à sa maison, A. Arthur Schiller a fait une faute d'anhistoricisme, une négation du contexte historique, un rejet pur et simple de l'histoire. Il a inventé cela idéologiquement, sans prendre en considération ce que nous savons du droit romain, le tout, en n'ayant aucune preuve pour son assertion, sinon l'éblouissement que procure toujours une analogie. C'est qu'on peut appeler de la fraude scientifique. 
Dans le « dossier Morton J. Horwitz » ${ }^{40}$, Alan Watson se penche sur un des grands noms de la recherche universitaire aux États-Unis, celui qui est censé avoir donné la version la plus juste et la plus adéquate de la "transformation du droit états-unien ${ }^{41}$. Alan Watson observe froidement que M. J. Horwitz s'est simplement fondé sur un a priori à la mode - à savoir sur le paradigme d'une surdétermination " politique " et sur la figuration d'un " jeu de pouvoir » (mots très populaires dans les années 1960 - 1970s, et même aujourd'hui) - et qu'il a négligé l'histoire concrète et réelle du droit ${ }^{42}$. Dans une analyse en détail du "riparien law ${ }^{43}$ - droit de l'eau - il démontre, par la doctrine autant que par la jurisprudence, qu'Horwitz n'arrive pas à prouver son a priori. Là où Horwitz croit fournir une révélation "réaliste " (sic !) de la transformation du droit états-unien, Watson ne constate qu'une histoire idéologique montée de toute pièce pour épater la galerie. Son jugement sur le livre de Horwitz affirme qu'il s'agit :

(..) d'une démonstration très sexy et, en tant que telle, immédiatement attractive. Qu'il ait été couronné de succès et qu'il ait eu une influence avec cette théorie, cela ne fait aucun doute. Ma difficulté, cependant, c'est qu'une théorie reposant sur des opinions concernant l'analyse juridique n'a aucune valeur si l'analyse n'est pas exacte. Je ne pense pas que l'analyse de Horwitz concernant les faits ou le droit ou les cas qu'il analyse est exacte. Je me suis même à l'occasion interrogé s'il avait lu les cas en question ${ }^{44}$.

D’où la question: une étude de l'histoire du droit états-unien fondée sur une lecture biaisée et idéologique peut-elle avoir de la valeur? Une étude de l'histoire qui repose sur une théorie surdéterminante ou simplement déterminante n'est-elle pas, immanquablement, frauduleuse?

Dans le "dossier Duncan Kennedy ${ }^{45}$, Alan Watson ne se montre guère plus charitable. Ici, Alain Watson analyse l'article controversé et postmoderne de Duncan Kennedy "The Structure of Blackstone's Commentaries " ${ }^{46}$ et il affirme, en toute simplicité, que " Cet article est de toute évidence clairement faux et ses 128 pages [sic !] sont incompréhensibles [brumeuses]» ${ }^{47}$. Pourquoi?

Déjà, dans les mots introductifs de l'article de Duncan Kennedy se révèle, indique Alan Watson, le fond du problème, car il prétend qu'il peut accéder directement à la " conscience » et à la " pensée juridique » de William Blackstone ! Comment fait-il cela ? Comment peut-il rationnellement effectuer une telle opération transmentale et transhistorique? Ne s'agit-il pas, plutôt, d'une mission impossible et illogique ? De toute évidence, Duncan Kennedy en est incapable. D'où la constatation d'Alan Watson que l'affirmation sert uniquement à prétendre, faussement, qu'il n'a, de ce fait, pas besoin d'analyser ce que William Blackstone a réellement écrit (à savoir le livre qu'il prétend analyser et qu'en conséquence il n'analyse pas) ni d'examiner ses sources (qu'il néglige, qu'il change et qu'il transfigure) ni même de considérer son contexte et l'histoire réelle (il affirme sans gêne que cela ne l'intéresse pas), car il travaille sur quelque chose que lui seul sait ! Duncan Kennedy ne travaille, en fait, que sur une " image à la mode » concernant 
une soi-disant tradition qu'il se forge en privé, une image de l'idéodroit qui permet de séduire et d'avoir des "followers" (c.-à-d. suiveurs), mais qui ne nous informe en rien (et plutôt nous désinforme, nous manipule) sur Blackstone.

En conséquence, à Alan Watson d'attester que cela n'a aucun sens ! À lui, également, de nous fournir, en 16 pages, une solide contre-analyse précise et critique de la "structure des Commentaires " de William Blackstone; de faire l'analyse qui n'existait pas chez Duncan Kennedy et qui ne pouvait pas exister chez lui non plus ${ }^{48}$.

La critique de collègues, de même que la distanciation intellectuelle à l'égard de leurs élucubrations et égarements idéologiques, pour ne rien ajouter quant à leurs divagations politiques, leurs fantasmes " poétiques " et "littéraires ", ont toujours été une question problématique, plutôt taboue, dans les facultés de droit $^{49}$. Le consensus a été de ne rien voir, de ne rien entendre, de ne rien dire et de ne jamais critiquer. La collégialité protège la médiocrité autant que la bêtise et la recherche mal faite. Le plus souvent, le silence a été la seule réponse des facultés à l'égard des brebis égarées et des surinvestissements idéologiques. Or, les trois professeurs (Abraham Arthur Schiller; Morton J. Horwitz; Duncan Kennedy) que critique Alan Watson représentent, sans conteste, la crème du jus-oligarchie des États-Unis. II s'agit de trois pontifes qui savent s'imposer et faire régner l'ordre, à leur avantage $\mathrm{e}^{50}$. Que Alan Watson les critique sans porter de gants peut uniquement se retourner contre lui et lui nuire considérablement. Et, pourtant, c'est le souci pour la qualité de la recherche et la vigilance critique contre l'inculture et la soumission idéologique, qui l'anime; - il n'aime pas les tricheurs universitaires et il veut que cela se sache.

Or, c'est en somme surtout au niveau iusfacultaire que cela prend entièrement tout son sens! Que dire d'un milieu incapable de sérieusement faire fi de la tricherie, de l'imposture et des constructions idéologiques ? Que dire d'un milieu qui reste muet sur ce qui n'a guère de sens logique et rationnel tant au niveau du droit que sur ce qui relève du fantasme idéologique propre à l'extrême droite, sinon à leurs partisans de la néo-gauche ou du courant radical-réactionnaire culturel ? Ne faut-il pas plutôt ouvrir les yeux et œuvrer en faveur d'un sens moderne à accorder à notre contemporanéité juridique ?

\section{Pour conclure quant à la honte et à sa guérison}

Alan Watson a choisi de briser la glace, il a osé exprimer ce qu'il pense et il l'a fait au profit de ce petit monde de l'enseignement juridique qu'il aime tant et qu'il a tant servi loyalement toute sa vie. La critique d'Alan Watson est un cri d'alarme, un cri universitaire contre la crétinisation galopante, contre la déculturation niaiseuse que privilégient nos élites, nos oligarchies de gauche et de droite. Ceux-ci se méfient, avec raison, d'une culture juridique qu'ils ne dominent pas et qui risque de porter préjudice à leurs privilèges, leurs autorités et à leurs " pouvoirs".

En lisant Alan Watson se discerne le message d'un homme ayant fait le choix de "rester debout ". Chaque phrase, chaque formule, chaque critique dans le livre d'Alan 
Watson, lue mais surtout comprise, témoigne du fait que nous avons terriblement tort de céder au chant des sirènes qui œuvre en faveur de l'irrationalisme, du pouvoir oligarchique, de l'Establishment et du " progressisme réactionnaire ». Eux n'aiment pas la culture juridique, ils préfèrent voir les dos se courber, ils aiment avoir le pouvoir sur autrui, sur vous, sur l'enseignement du droit, sur l'individu qui cherche à obtenir justice.

Affirmons-le sans ambiguïté ! Nous sommes d'accord avec Alan Watson et avec son diagnostic de la culture iusfacultaire nord-américaine. II a vu juste et il a raison. II a honnêtement rendu un immense service à la communauté juridique. Et il a surtout bien compris les fêlures contemporaines qui corrompent le monde iusfacultaire et qui risquent de s'élargir de plus en plus au profit de l'idéodroit, de l'ignorance et de l'irrationalité tant juridique, que politique et sociale. Il a surtout compris la corruption lancinante à l'œuvre, à l'intérieur des iusfacultés et comment tout ceci porte déjà préjudice à la culture juridique et risque de tourner en catastrophe. Et il a notamment bien compris que cela ne va que s'empirer et s'envenimer sans qu'il n'y ait aucune cure miracle à portée de main !

Si la lucidité critique d'Alan Watson se focalise, le titre l'indique, sur la banqueroute de l'enseignement du droit aux États-Unis, il n'y a aucune raison de se réjouir ailleurs. L'idéologie que privilégient nos oligarchies de droite et à gauche ne fera pas de quartier, et voilà ce qui attend le petit monde iusfacultaire partout dans le monde si personne ne proteste, si personne ne critique et si personne ne défend avec vigueur un enseignement du droit de qualité, un enseignement qui peut aussi se justifier au niveau de l'intelligence juridique. Hélas, aujourd'hui, c'est indubitablement les intérêts du crétinisme « de l'idéodroit " qui sont sur le haut du pavé et qui s'imposent, de plus en plus, et toujours à nos dépens ${ }^{51}$. C'est le crétinisme de l'idéodroit qui vaincra, en cherchant à mettre le grappin sur le curriculum iusfacultaire pour leur gloire, leur pouvoir, leur business. Tant pis pour le droit! Tant pis pour les étudiants. Tant pis pour l'enseignement d'excellence et tant pis pour un iusfaculté de qualité !

À ce moment-là, convoquons l'aphorisme si lucide que nous avais apporté Georg Christoph Lichtenberg en 1793 : « Aujourd'hui on cherche partout à répandre le savoir, qui sait si dans quelques siècles il n'y aura pas des universités pour rétablir l'ancienne ignorance ${ }^{52}$.

Hélas, c'est vrai ! II y a aujourd'hui des universités et des universitaires qui ne font que cela : rependre l'ancienne pour en faire la nouvelle ignorance, en exigeant que tout le monde fasse de même. Le problème réside dans le fait que ces universités et ces universitaires répandent, en ce moment même, l'ancienne ignorance en redoublant d'efforts pour y ajouter encore plus d'ignorance. Hélas, de plus en plus d'iusfacultés agissent de cette manière, en s'engageant dans l'ignorance, dans l'irrationalité et dans les fantasmes idéologiques et, surtout, en abusant toujours plus du mot " droit ». Ils abusent du mot « droit " pour célébrer l'idéodroit (ou mieux: l'idiot-droit) de leurs imaginations, de leurs lectures, de leurs idéologies et de leurs jeux de pouvoir. Tous célèbrent l'ignorance juridique en s'imaginant être capables de dire "vrai » et de "dire droit ", et tant pis pour la vérité. On ne parle plus d'une exigence d'un « droit sain » qui n'entre certainement pas dans leur agenda si bien garni. 
Pour tout ceci, il faut lire Allan Watson et " Honte à l'enseignement juridique américain ", car il n'est jamais trop tard pour dire " non » à la situation présente et, comme lui, se tenir debout dans la construction d'un « droit sain » par des enseignants qui connaissent la matière et savent établir une réelle relation avec leurs étudiants, sur le modèle de Allan Watson!

\footnotetext{
*Professeur, Université Laval, Québec ; bjarne.melkevik@fd.ulaval.ca.
}

${ }^{1}$ Alan Watson (i.e. William Alexander Jardine Watson), The Shame of American Legal Education, Lake Mary (FL), Vandeplas Publishing, (2005 $1^{\text {re }}$ édition), 2006, 2e edition. Alan Watson souligne, page xxii, qu'il s'appuie sur des arguments développer ultérieurement dans deux articles: idem, "Legal Education Reform: Modest Suggestions", Journal of Legal Education, Vol. 51, No. 1, March 2001, p. 91 - 97; et idem, "Introduction to Law for Second-Year Students?", Journal of Legal Education, Vol. 46, No. 3 (September 1996), p. 430 - 444. Cf. également, Alan Watson, Law out of context, Athens (Georgia) \& London, University of Georgia Press, 2000, pages 140 - 163.

2 En ce qui concerne l'exportation et l'importation du modèle de law-school, cela se réalise à présent déjà dans l'Europe de l'Est, au Moyen-Orient, en Chine, en Amérique latine, etc. Nous n'analyserons pas ce phénomène.

${ }^{3}$ Alan Watson a publié de nombreux titres, mentionnons uniquement : "Legal Transplants: An Approach to Comparative Law" (1974), "Society and Legal Change" (1977), "Jesus and the Jews: The Pharisaic Tradition in John" (1995), "Ancient Law and Modern Understanding: At the Edges" (1998), "Sources of Law, Legal Change, and Ambiguity" (2d ed., 1998), "The Evolution of Western Private Law" (2000), "Legal History and a Common Law for Europe" (2001), "Authority of Law; and Law" (2003).

${ }^{4}$ Bjarne Melkevik, "Scolies sur l'avenir des facultés de droit ", dans, idem, Philosophie du jugement juridique, Ste-Foy/Québec, Les Presses de l'Université Laval, coll. Diké, 2010, p 173 - 183 (repris dans idem, Philosophie du droit. Volume 2, Ste-Foy, Les Presses de l'Université Laval, coll. Diké, 2014, p 191 -202. Voir aussi, idem, "Critiques d'un texte mythique : "Réflexions sur l'enseignement du droit et la reproduction des hiérarchies. Une polémique autour du système" de Duncan Kennedy ", dans Studia Universitatis Babes-Bolyai (Cluj-Romanie), anul LIV, janvier 2011, no 1, p 99-128.

${ }^{5}$ Bjarne Melkevik, "Quatre thèses critiques sur l'interdisciplinarité dans le domaine du juridique. Une précision d'épistémologie juridique ", dans Jean-Jacques Sueur et Sarah Farhi (dir.), Pratique(s) et enseignement du droit » - L'épreuve du réel, Actes du colloque organisé les 22 et 23 avril 2015 à la Faculté de droit de l'université de Toulon, Paris, Lextenso, coll. Contextes-Culture du droit, 2016, p. 354 - 372.

${ }^{6}$ Cf. William Shakespeare, Hamlet (I, 4, Marcellus), traduction François-Victor Hugo, Paris, Pagnerre, 1859-1866 : " Il y a quelque chose de pourri au royaume du Danemark. »

7 Alan Watson est surtout connu pour son livre, Legal Transplants: An Approach to Comparative Law, Edinburgh, Scottish Academic Press, 1974; reprint edition, University of Georgia Press 1993. Le mot "legal transplants" n'a pas d'équivalence directe en langue française. Une traduction convenable serait " greffes législatifs " ou " greffes juridiques ", " greffes juris-dicton ", tandis qu'" infusion législative ", " contamination législative ", " import législatif », etc. peut être défendu. L'idée de base c'est qu'un système de juridiction s'inspire d'un autre système de juridiction pour des réformes législatives, des constructions doctrinales ou " jurisprudentielles ", ce que démontre que le phénomène de " legal transplants » peut se faire, se réaliser, dans différentes manières selon les contextes, les objectifs et les circonstances particulières propres à chaque système de juridiction. La littérature sur « legal transplants » est immense ; mentionnons uniquement John W. Cairns, Codification, Transplants and History : Law Reform in Louisiana (1808) and Québec (1866), Clark (New Jersey), Talbot Publishing (The Lawbook Exchange Ltd), 2015. 
${ }^{8}$ Avec le nombre très élevé de law-schools aux États-Unis, il va sans dire qu'il existe des exceptions et même des exceptions valorisant l'enseignement d'excellence qu'Alan Watson privilégie. Notons que les law-schools sont accrédités sur le niveau interétatique par American Bar Association (Le Barreau des États-Unis) et 203 parmi eux ont acquis ce statut aujourd'hui. Or, le Barreau des chaque État aux États-Unis peut avoir leur propre système d'accréditation d'un law-school pour préparation de leur examen de pratique. Il existe également un grand nombre de "law schools " qui ne sont pas accréditées ni sur le niveau interétatique ni sur le niveau des États. Et il existe aussi de " law schools » par " correspondances » ou par " internet études ». II est en plus possible, dans plusieurs états, de devenir avocat sans passer formellement par un " law-school »; soit par l'apprentissage pratique (auprès d'un juge ou avocat) ou par la réussite de l'Examen de pratique (c.-à-d. en Vermont, Washington, Californie, Virginie et Wyoming). Cf. American Bar Association, Section of Legal Education and Admissions to the Bar, ABA Standards for Approval of Law Schools and Interpretations, Chicago, American Bar Association (ABA), 1996.

${ }^{9}$ Alan Watson, The Shame of American Legal Education, op. cit., page $43-78$.

${ }^{10}$ Voir Bruce A. Kimball, The Inception of Modern Professional Education. C. C. Langdell, 1826 - 1906, Chapel Hill (N.C), University of North Carolina Press, 2009.

${ }^{11}$ Alan Watson se réfère à Robert Stevens, Law School: Legal Education in America from the 1859 to the 1980s, Chapel Hill (N.C.), University of North Carolina Press, 1983; et à William P. LaPiana, Logic and Experience: The Origin of Modern Legal Education, New York, Oxford University Press, 1994.

${ }^{12}$ Alan Watson, The Shame of American Legal Education, op. cit., page 68. Notre traduction: "It was introduced by Christopher Columbus Langdell, dean of Harvard Law School, who believed that law was a science, and should be taught in a scientific method from cases. (...) The result is absurd. But tradition, once established, is not easily dislodged."

13 Hans Kelsen, Théorie pure du droit. Introduction à la science du droit, Neuchâtel, Éditions de la Baconnière, coll. Être et Penser no 37, 1953, page 117 - 122. Cf. Carlos Miguel Herrera, La philosophie du droit de Hans Kelsen. Une introduction, Québec, Presses de l'Université Laval, coll. Diké, 2004.

${ }^{14}$ Voir, Richard A. Posner, The Problems of Jurisprudence, Cambridge (Mass.), Harvard University Press, 1990, page 14 - 15: "Into the breach stepped the American legal formalists, of whom the best known today is Dean Christopher Columbus Langdell of the Harvard Law School. On one level the formalist were Platonists, believing that there existed a handful of permanent, unchanging, indispensable principles of law imperfectly embodied in the many thousands of published judicial opinions, and that the goal of legal reasoning was to penetrate the opinions to the principles. That is the flavor of Langdell's own scholarship." Cf. également, Richard A. Posner, "Legal Formalism, Legal Realism, and the Interpretation of Statutes and the Constitution", dans Case Western Reserve Law Review, vol. 37, 1986, p $179-217$.

${ }^{15}$ En suivant le proverbe de classicisme juridique: « Res judicata pro veritate accipitur (ou habetur) » (La chose jugée est acceptée ou tenue pour vérité ou vraie/ La chose jugée est tenue pour vrai). Voir: Dig. 50, 17, 207.

${ }^{16}$ Voir, Stephan M. Feldman, American Legal Thought from Premodernism to Postmodernism, New York \& Oxford, Oxford University Press, 2000, p 94: "Hence, two central features of Langdellian legal science were a positivist focus on the decided cases and the use of inductive reason to discover legal principles"

${ }^{17}$ Aristote, Rhétorique 1, 1357a, et 1395b 22.

${ }^{18}$ Voir le problème (et critique) de l'induction chez David Hume (1711 -1776), Traité de la nature humaine $(1738-1740)$; plusieurs éditions. 
${ }^{19}$ Voir, Bjarne Melkevik, Épistémologie juridique et déjà-droit, Paris, Buenos Books International, 2014.

${ }^{20}$ Alan Watson, The Shame of American Legal Education, op. cit., page $79-88$.

${ }^{21} \mathrm{Cf}$. Matthieu, chapitre 14, versets 14 à 21, idem 15, 32-38; Marc 6, 34-44, idem 8, 1-9; Luc 9, 12-17; Jean 6, 5-14.

${ }^{22}$ Alan Watson, The Shame of American Legal Education, op. cit., p 1 - 24.

${ }^{23}$ Alan Watson, The Shame of American Legal Education, op. cit., page $\mathrm{xx}$ - xxi. Notre traduction de: "The so-called Socratic Method of teaching - a misnomer, actually - is endemic in the U.S. (...) Those who use it - the vast majority of law professors - believe it hones analytical skills. To a great degree I disagree: it is teaching by terror. The professor picks, usually apparently at random, a student whom she questions about an assigned case. The student can seldom answer satisfactorily. »

${ }^{24}$ Alan Watson, The Shame of American Legal Education, op. cit., page 46 - 47, et page $48-51$ ("Teaching by Terror"), cf. p $71-72$.

${ }^{25}$ Pour une description, vue à partir de l'étudiant, de cette anti-pédagogie, voir, Scott Turow, One L. The Turbulent True Story of a First Year at Harvard Law School, 1977 (avec une Postface, 2010). II n'y a pas de différence entre la 1re édition (1977) et la troisième (2010), sauf en ce qui concerne le Postface.

${ }^{26}$ Alan Watson, The Shame of American Legal Education, op. cit., page 68: Notre traduction: "But just imagine a college physics course where only a few isolated experiments are studied, where the relationship of one to the other is not set out, where the theoretical underpinnings are not stated, and where virtually all of the writings of the scholars are ignored. The result is absurd".

${ }^{27}$ Alan Watson, The Shame of American Legal Education, op. cit., p $20-21$, cf. $79-88$.

${ }^{28}$ Alan Watson, The Shame of American Legal education, op. cit., page xvii et xviii: Notre traduction de "The American casebook approach (....) is confusing and uninformative. Individual cases are abridged and torn out of their contexts. Students are not informed whether the case is central to the law or is at the edges. Nor are they given an outline of the main principles of the subject. Since this is the standard approach from the first day of law school, first year students are paranoid. Second-year students are bored, and are recognized to be so by almost all law professors".

${ }^{29}$ Alan Watson, The Shame of American Legal education, op. cit., page $79-88$.

${ }^{30}$ Ce qui était - sur le niveau universitaire et historique - le résultat de la révolution humboldtienne (c.-à-d. Friedrich Wilhelm Christian Karl Ferdinand, Freiherr von Humboldt, plus connu sous le nom Wilhelm von Humboldt, et en français, Guillaume de Humboldt, 1767 - 1835), de l'enseignement universitaire et surtout du droit. Von Humboldt en réformant la nouvelle Université de Berlin (Alma Mater Berolinensis en 1810) a exigé que chaque professeur enseigne les résultats de leurs propres recherches. La révolution européenne quant à la "doctrine du droit » est largement redevable à l'enseignement universitaire à partir de la recherche individuelle des professeurs en droit. L'obligation des professeurs d'université d'enseigner "à partir de leurs propres recherches" a bien sûr des racines culturelle et historique - surtout sur le niveau individuel - profondes dans la civilisation " occidentale ». Alan Watson, dans The Shame of American Legal Education, revient à plusieurs reprises sur comment certains professeurs avec leurs « livres de doctrine » a eu une influence marquant et bénéfique quant au développement de la culture juridique européenne.

${ }^{31}$ Bjarne Melkevik, "Quatre thèses critiques sur l'interdisciplinarité dans le domaine du juridique. Une précision d'épistémologie juridique ", dans Jean-Jacques Sueur et Sarah Farhi-Segond (dir.), Pratique(s) et enseignement du droit »-L'épreuve du réel, Actes du colloque organisé les 22 et 23 avril 2015 à la Faculté de droit de l'université de Toulon, Paris, Lextenso, coll. Contextes-Culture du droit, 2016, p $354-372$, spécialement la section 3 : « Contre la « littérarisation » du domaine juridique ». 
${ }^{32}$ Alan Watson, The Shame of American Legal Education, op. cit., p 79-88.

${ }^{33}$ Alan Watson, The Shame of American Legal Education, pages 9 - 10; notre traduction de "The casebook method of teaching is, in fact, an exercise in futility. It is the students themselves who are expected to build up a picture of law from the few generally disconnected scraps available to them and with virtually no tools. Students are left to guess what the editors' view of the law is rather than getting to what the law is all about. Instead of looking at the reasoning of the case in the light of the developed conceptual thought that preceded it, and of its place in a structured web of reasoned principle, they are provided in the first place with a single instance that justifies itself only by reference to particular features, leaving much to be understood. Much of importance to the case is left unsupported and unsaid because it rests on established principle. The students study, as it were, the study of grain of sand by walking around inside the grain and without reference to the rest of the beach, the surf and the sky. The problem is compounded when the discussion is limited largely to the concept - "Is it fair?"

${ }^{34}$ Alan Watson, The Shame of American Legal Education, idem, p. xx - xxi, notre traduction de: "My point is emphatically not that learning the law would be much easier - it obviously would be - if concepts and rules as they had developed in sores of cases were set out briefly, then were followed by discussion of a few cases choses to illustrate the rules, their parameters, and issues raised by borderline situations; and that this is something necessarily desirable. This is, perhaps, a virtue as some of colleagues think in making first-year law study unnecessarily difficult. My point is that the standard approach misrepresents the way law is, how it develops, and its relation to society. Concepts and principles are badly downplayed. So are rules, and their authority and stability. Cases are removed from their legally relevant context and the authority of the context is dismantled. Oddly perhaps, a further result of this approach is that often cases are removed from their social context. (...) The absence of theoretical underpinnings is a fatal flaw in the casebook approach. »

${ }^{35}$ Alan Watson, "The Shame of American Legal Education", op. cit., p 17: « The professors too, have been badly educated $"$.

${ }^{36}$ Alan Watson, "The Shame of American Legal Education ", op. cit., p $143-192$.

${ }^{37}$ Abraham Arthur Schiller, 1897 - 1977, était professeur à la Faculté de droit de l'Université Colombia de 1928 à 1977. En droit romain il a publié, Texts and Commentary for Study of Roman Law. Mechanisms and Development, New York 1946; et, Roman Law: Mechanisms of Development, Den Haag / New York 1978.

${ }^{38}$ Alan Watson, "The Shame of American Legal Education ", op. cit., p $143-152$. Les pages reprend, en abrégé, article de Alan Watson, "Trade Secrets and Roman Law: The Myth Exploded ", Tulane European \& Civil Law Forum, 1996, p 19- 29.

${ }^{39}$ A. Arthur Schiller, "Trade Secrets and Roman Law: the Actio Servi Corrupti », Columbia Law Review, vol. 30, 1929-1930, p 837 - 845. Notons que "action servi corrupti" (i.e. action préventive ou réparatrice contre la corruption d'un esclave ou d'une esclave), se rapport au : Institutiones 4, 6, 23; Digest 11, 3; Codex lustinianus 6, 2.

${ }^{40}$ Alan Watson, The Shame of American Legal Education, op. cit., p 152 - 176. Alan Watson reprend ici des arguments de son article "The Transformation of American Property Law: A Comparative Law Approach" Georgia Law Review, Vol. 24, No. 2 (Winter 1990), pp. 163-221. Le titre fait directement référence au Morton J. Horwitz, The Transformation of American Law, 1780-1860, Cambridge (Mass.) Harvard University Press, 1977.

${ }^{41}$ C'est le livre de Morton J. Horwitz, The Transformation of American Law, 1780-1860, Cambridge (Mass.) Harvard University Press, 1977, qui est l'objet du critique. Morton J. Horwitz est aussi de: The Transformation of American Law, 1870-1960: The Crisis of Legal Orthodoxy, New York, Oxford University Press, 1992, et de: The Warren Court and the Pursuit of Justice, New York, Hill \& Wang Publishing, 1998. 
${ }^{42}$ Le livre de Horwitz se compare avec analyse de William M. Wiecek, The Lost World of Classical Legal Thought: Law and Ideology in America, 1886 - 1937, New York, Oxford University Press, 1998. Notons pourtant que la discussion de Wiecek sur la montée et la chute du "classicisme " peut être plus éclairante comme une préhistoire de la Cour suprême des États-Unis que comme une description du « droit » de la fin du XIXe et au début du vingtième (ou la pensée juridique en général).

${ }^{43}$ Le mot "riparian law" n'a pas de vrai équivalent dans la langue française (et non plus dans le droit français - et québécois - d'aujourd'hui). Il est tradition de le traduire en "droit à l'eau ", même si une telle traduction est trop restrictive. Le mot « riparian » se réfère au " riverain » (et ne nécessite donc pas un droit de propriété, stricto sensu) et peut en conséquence se référer autant à l'accès à l'eau (pour approvisionnement et consommation) que de pêche, de l'installation de ponton (et un système d'accotement), de transport fluvial, de navigation, de chasse, d'installation de systèmes de protection de berges, et ainsi de suite.

${ }^{44}$ Alan Watson, The Shame of American Legal Education, op. cit., p 176. Notre traduction de: "(...) a very sexy thesis, and as such immediately attractive. That he has been successful and influential with this theory there can be no doubt. My difficulty, though, is that a theory that is based on judgement about legal analysis has no value unless the analysis is accurate. I do not think Horwitz' analysis of the facts or law or both of the cases his discusses is accurate. I have at times even wondered if he has read the cases."

${ }^{45}$ Alan Watson, The Shame of American Legal Education, op. cit., p 176 - 192, cf. 120-126. Alan Watson se réfère et reprend l'argumentation développée dans son article "The Structure of Blackstone's Commentaries", Yale Law Journal 97 (1988), p 795 - 821.

${ }^{46}$ Duncan Kennedy, "The Structure of Blackstone's Commentaries", 28 Buffalo Law Review, 1979, p 209 - 382. Duncan Kennedy fait dans son article semblant de présenter une analyse de William Blackstone's Commentaries on the Laws of England (1765 - 1796). Le livre de Blackstone est republié plusieurs fois ; il existe trois traductions historiques en langue française.

${ }^{47}$ Alan Watson, The Shame of American Legal Education, op. cit., p 176. Notre traduction de: "This article is obviously wrong on its face and its 128 pages [sic!] are incomprehensible". Notons qu'Alan Watson écrit ici "128 pages", tandis que l'article de Duncan Kennedy est de facto 174 pages. Cf. également le jugement d'Alan Watson, id, page 191 - «I insist that Kennedy's paper is without merit. »

${ }^{48}$ Voir notre critique de Duncan Kennedy, dans Bjarne Melkevik, "Critiques d'un texte mythique : Réflexions sur " L'enseignement du droit et la reproduction des hiérarchies. Une polémique autour du système " de Duncan Kennedy ", Studia Universitatis Babes-Bolyai : Iurisprudentia, anul LIV, janvier 2011, no 1, p 99 - 128. L'article critique "les mises en image discursives » que construit Duncan Kennedy concernant sa conception privée de l'idéodroit. II s'agit d'une critique d'une " critique qui ne l'est pas » puisqu'en soumettant le texte mythique de Duncan Kennedy « L'enseignement du droit et la reproduction des hiérarchies, à une relecture attentive et critique, c'est une image démagogique et sophiste qui se dégage. Ce texte légendaire, qui pendant des décennies a servi de justification à une politique étriquée de "rectitude politique » dans les facultés de droit, n'est en fin de compte qu'un sursaut contre-culturel sans contenu véritable. L'article analyse soigneusement l'utilisation que Duncan Kennedy fait du mot " hiérarchie " pour démontrer comment ce mot est construit en tant qu'un " attrape-mouche » pour capter l'insécurité et le désarroi des étudiants. Soumettant le modèle contreculturel de Duncan Kennedy et sa politique de pouvoir à une critique, l'article insiste sur l'impasse aussi bien politique que démocratique qui s'en dégage. La critique débouche sur la proposition qu'il vaut mieux penser le monde jus facultaire selon des critères démocratiques et à considérer la vocation d'enseigner dans le contexte d'un droit moderne et démocratique. 
${ }^{49}$ Voir, Boris Barraud, La recherche juridique. Science et pensées du droit, Paris, L'Harmattan, coll. Logiques Juridiques, 2016, p $520-523$.

50 Voir, Alan Watson, The Shame of American Legal Education, page 192, sur Duncan Kennedy: " To win approval and a suitable position it was important to show allegiance to Kennedy. "

51 Voir, Christophe Jamin, La cuisine du droit. L'école de droit de Science Po : une expérience française, Paris, L.G.D.J., coll. Forum, 2012. Et le Collectif, Numéro thématique, "L'enseignement du droit au début du 21e siècle. Perspectives critiques ", Jurisprudence. Revue critique, Chambéry (France), où il n'y a aucun article critique du tout et nonobstant ce qu'annonce - de façon non critique ? - le titre.

52 Georg Christoph Lichtenberg, Le Miroir de l'âme (1793-1796), (trad. Charles Le Blanc), Paris, Édition José Corti, coll. Domaine Romantique, 1997, p. 504. 